\title{
USE OF HAND DIAGRAMS IN SCREENING FOR ULNAR NEUROPATHY: COMPARISON WITH ELECTRODIAGNOSTIC STUDIES
}

\author{
ROBERT A. WERNER, MD, ${ }^{1,2,3}$ TONY CHIODO, MD, ${ }^{2}$ TERESA SPIEGELBERG, BS, ${ }^{2}$ and ALFRED FRANZBLAU, MD ${ }^{3}$ \\ ${ }^{1}$ Department of Physical Medicine and Rehabilitation, Veterans Administration Health System, \\ 2215 Fuller Road (117), Ann Arbor, Michigan 48105, USA \\ ${ }^{2}$ Department of Physical Medicine and Rehabilitation, University of Michigan Health Systems, Ann Arbor, Michigan, USA \\ ${ }^{3}$ Department of Environmental Health Sciences, School of Public Health, University of Michigan, Ann Arbor, Michigan, USA \\ Accepted 1 May 2012
}

ABSTRACT: Introduction: The objective of this study was to determine whether a hand diagram could be used to predict ulnar mononeuropathy. Methods: This was a prospective study of 117 consecutive patients referred for hand symptoms. Each subject filled out a hand diagram of symptoms and had median and ulnar sensory and motor nerve conduction studies, including ulnar conduction across the elbow. Results: The best model for predicting an ulnar mononeuropathy included hand diagram scores of definite or possible. The model had a sensitivity of $50 \%$ and specificity of $93 \%$ with an ROC area of 0.90 . Conclusions: The ulnar hand diagram scoring system can be useful as a screening tool in the electrodiagnostic laboratory or for epidemiologic studies.

Muscle Nerve 46: 891-894, 2012

Unar neuropathy (UN) is a common nerve entrapment disorder of the upper extremity. Typical symptoms include numbness, tingling, or burning sensation in the fourth and fifth digits of the hand. The most common site of entrapment is the elbow, although entrapment at the wrist is also a possibility.

$\mathrm{UN}$ at the elbow is the second most common compression neuropathy of peripheral nerves. Although carpal tunnel syndrome (CTS) is the most common nerve entrapment, $\mathrm{UN}$ at the elbow has an annual incidence that is approximately onethirteenth that of CTS, or 24.7 per 100,000 population. ${ }^{1}$ Another study estimated the prevalence rate at $0.6-0.8 \% .^{2}$ Repetitive work is considered a risk factor. ${ }^{3}$ Specifically, repetitive flexing and extending of the elbow is considered to be a risk factor. $\mathrm{UN}$ at the elbow is more common in men. ${ }^{4,5}$ Sustained pressure over the elbow region is also a risk factor. There has been little study regarding the incidence or prevalence of this disorder in the working population, although one study of female floor cleaners demonstrated a prevalence of $7 \% .^{6}$ There are no general population estimates of the prevalence of UN at the wrist.

The diagnosis of UN at the elbow or Guyon canal nerve entrapment is typically made by clini-

Abbreviations: AANEM, American Association of Neuromuscular and Electrodiagnostic Medicine; CTS, carpal tunnel syndrome; CV, conduction velocity; EMG, electromyography; ROC, receiver operator curve; TOS, thoracic outlet syndrome; UN, ulnar neuropathy

Key words: electrodiagnostic test, epidemiology, nerve conduction studies, screening, sensory nerve function, ulnar nerve

Correspondence to: R. A. Werner; e-mail: rawerner@umich.edu

(C) 2012 Wiley Periodicals, Inc.

Published online 5 October 2012 in Wiley Online Library (wileyonlinelibrary. com). DOl 10.1002/mus.23452

Hand Diagrams for Ulnar Neuropathy cal presentation and confirmed by electrodiagnostic testing. Clinically, an affected patient complains of numbness and tingling in an ulnar distribution. In more severe cases there is weakness and atrophy of ulnar innervated hand intrinsic muscles. There is a self-administered questionnaire that can be used to evaluate symptom severity (similar to the Boston questionnaire for CTS), but it has not been used to assess the prevalence of UN in the general population. ${ }^{7}$

In patients with an $\mathrm{UN}$, as in patients with CTS, there is significant variability in the distribution of reported symptoms versus the anatomical distribution of the sensory fibers. Hand diagrams have been used reliably for evaluation of patients suspected of having CTS. ${ }^{8,9}$ Hand diagrams have been shown to have a sensitivity of $80 \%$ and specificity of $90 \%$ for evaluation of patients with suspected CTS, but they had not yet been used for the evaluation of an UN. Thus, our aim was to establish and test a simple self-administered hand diagram for the evaluation of an UN. The instrument we developed was intended for use in an office practice, but it also has application for population screening. We understand that there are other neurological lesions that can present with a similar sensory distribution. A C8 radiculopathy, lower trunk brachial plexopathy, or thoracic outlet syndrome (TOS) can also present with symptoms in the fourth and fifth digits of the involved hand.

In this prospective study we utilized a hand diagram for all patients referred to the electrodiagnostic laboratory with hand symptoms. We tested the sensitivity and specificity of this self-administered hand diagram for establishing a diagnosis of ulnar mononeuropathy.

\section{METHODS}

A total of 117 consecutive patients seen at the University of Michigan Electrodiagnostic Laboratory for upper extremity evaluation of hand symptoms were given a hand diagram to fill out independent of the electromyographic (EMG) evaluation. All subjects signed a consent form that was approved by the institutional review board of the University of Michigan. The hand diagram was rated for the possibility of an ulnar mononeuropathy using the 


\begin{tabular}{|c|c|c|}
\hline 2 & Definite & $\begin{array}{l}\text { Palmar symptoms in the fifth digit OR symptoms in the fifth digit and ulnar side } \\
\text { of hand. Symptoms may also be in the fourth digit BUT cannot include digits } \\
1,2 \text {, or } 3 \text {, nor the radial aspect of the palm. }\end{array}$ \\
\hline 1 & Possible & $\begin{array}{l}\text { Symptoms in the fifth digit and ulnar side of hand. Symptoms may also be } \\
\text { noted in the rest of the palm. Symptoms may be in the following digits: } \\
1,2,4,5 ; 1,3,4,5 \text {; or } 1,2,3,5 \text {. }\end{array}$ \\
\hline
\end{tabular}

classification protocol described in Table 1. An example of the hand diagram is shown in Figure 1.

All participants had nerve conduction testing that included at least the following studies: median and ulnar sensory nerve conduction studies at the wrist $(14 \mathrm{~cm}$, antidromic stimulation in digits 2 and 5) and median and ulnar motor nerve conduction studies with ulnar conduction across the elbow, as well as needle EMG evaluation of the upper extremity. Additional nerve conduction testing for an UN at the wrist (dorsal ulnar cutaneous nerve) and for CTS (midpalmar median and ulnar nerves) was added as needed at the discretion of the electromyographer. The electrodiagnostic consultant, who was blinded to the findings on the hand diagram, made the determination of a final diagnosis for each subject. The criteria followed AANEM guidelines for defining an ulnar mononeuropathy, including an absolute across-elbow conduction velocity (CV) of $<50 \mathrm{~m} / \mathrm{s}$ or an acrosselbow CV $>10 \mathrm{~m} / \mathrm{s}$ slower than in the forearm segment. ${ }^{10}$ An UN at the elbow was also diagnosed if there was conduction block (a 20\% drop in amplitude), or denervation restricted to the ulnar distribution. An UN at the wrist was diagnosed if there was an isolated abnormality of the ulnar sensory or motor nerve at the wrist (with a normal dorsal ulnar cutaneous response), or if the needle examination demonstrated denervation only in ulnar hand intrinsic muscles. In the case of isolated denervation in the ulnar innervated hand intrinsic muscles, without slowing or conduction block at the elbow, it was recognized that the lesion could still be at the elbow, but this was reported as an $\mathrm{UN}$ at or above the wrist. We acknowledge that lesions at the Guyon canal, which spare the sensory fibers, may not be recognized, because ulnar motor nerve conduction to the first dorsal interosseous muscle was not routinely tested.

Statistical analysis included a logistical regression to model which risk factors were predictive of finding of an UN. The electrodiagnostic criteria for an UN were used as the dependent variable, and hand diagram score, age, gender, height, and weight were the independent variables. Stata soft- ware (StataCorp, College Station, Texas) was used for all analyses, including estimates of sensitivity and specificity and generation of the ROC graph. The ROC graph is based on the logistic regression model described earlier.

\section{RESULTS}

A total of 117 subjects were recruited in the first part of the study. The mean age was $42.9 \pm 12.4$ (range 20-92) years. Fifteen percent were male. The mean height was $1.66 \mathrm{~m}$, with a mean weight of $84.3 \mathrm{~kg}$. A total of 22 subjects had a classification of possible or definite UN using the hand diagram criteria. Twenty subjects were classified as having electrodiagnostic criteria for an UN (17 at the elbow and 3 at the wrist or above). Of the remaining 97 subjects, 43 had normal studies, 52 had a median neuropathy at the wrist, 1 had a median neuropathy at the elbow, and 1 had a C5 radiculopathy.
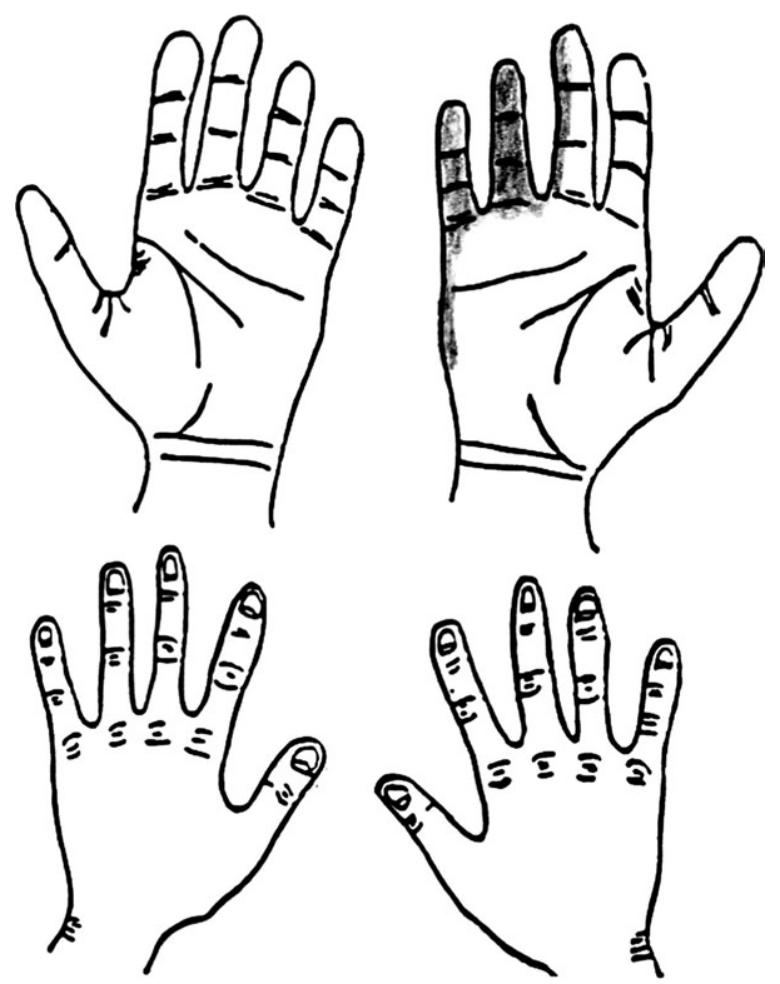

FIGURE 1. Typical hand diagram with symptoms in the right ulnar nerve distribution. 
Table 2. Results of logistic regression analysis using electrodiagnostic criteria for UN as dependent variable, and hand diagram score, age, gender, height, and weight as independent variables.

\begin{tabular}{lcccl}
\hline & Odds ratio & $Z$ & $P>|z|$ & $95 \% \mathrm{Cl}$ \\
\hline Hand diagram & 9.5 & 3.20 & 0.001 & $2.4-37.8$ \\
Age & 1.1 & 2.56 & 0.01 & $1.0-1.1$ \\
Gender & 3.4 & 1.34 & 0.18 & $0.6-19.9$ \\
Weight $(\mathrm{kg})$ & 1.0 & -1.43 & 0.16 & $0.9-1.0$ \\
Height $(\mathrm{cm})$ & 1.2 & 2.30 & 0.02 & $1.0-1.3$ \\
\hline
\end{tabular}

$N=115 ;$ chi-square $=42.57 ; \log$ likelihood $=-31.85 ;$ pseudo- $R^{2}=$ 0.40 .

When modeling the finding of an ulnar mononeuropathy using the electrodiagnostic criteria as the "gold standard," we evaluated the predictive value of the hand diagram criteria for an UN. We controlled for age, gender, height, and weight in the model. The best model included hand diagram scores of 1 or 2 (definite or possible) (Table 2). The finding of a positive hand diagram had an odds ratio of 9.5 [95\% confidence interval (CI) 2.4-37.8]. Age was significant in the model with an odds ratio of 1.06 (95\% CI: 1.02-1.12) for each additional year of life. Taller individuals were also at higher risk; the odds ratio was 1.2 (95\% CI 1.21.3) for each additional centimeter in height. Gender and weight were not significant in the model. The model had a sensitivity of $50 \%$ and a specificity of $93 \%$ with an ROC area of 0.90 (point A in Fig. 2, also see Table 3). According to the ROC figure, the cut-off for defining an ulnar mononeuropathy could be adjusted to yield a sensitivity of $80 \%$ and a specificity of $85 \%$ (point B in Fig. 2). When body mass index (BMI) was substituted for height and weight in the model, the variable was not significant. Two subjects did not provide a weight

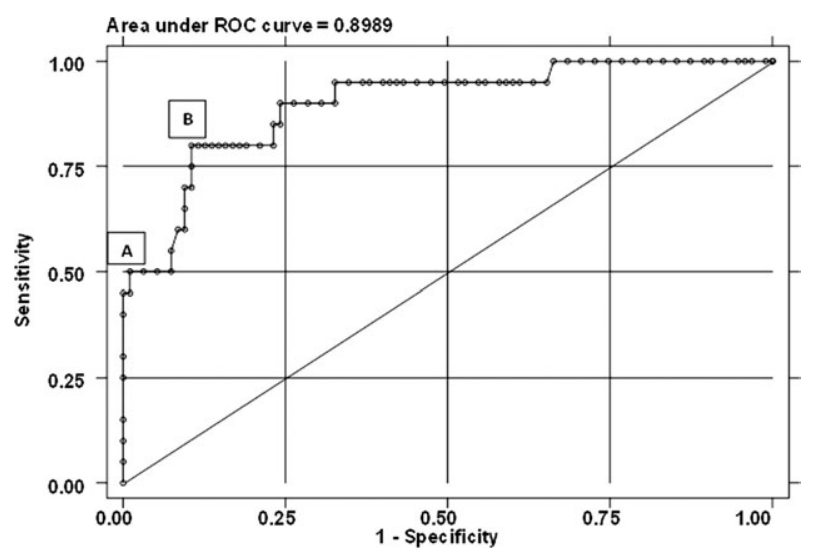

FIGURE 2. Receiver operator curve demonstrates the sensitivity and specificity of the model for ulnar mononeuropathy, which includes the ulnar hand diagram, age, gender, height, and weight as independent variables. The boxes with (A) and (B) represent alternative cut-off points for defining sensitivity and specificity.
Table 3. Sensitivity and specificity from logistic regression model for UN using hand diagram (grades 1 or 2), age, gender, height, and weight as predictor variables.

\begin{tabular}{lccc}
\hline & $\begin{array}{c}\text { NCS/EMG } \\
\text { positive } \\
\text { for UN }\end{array}$ & $\begin{array}{c}\text { NCS/EMG } \\
\text { negative } \\
\text { for UN }\end{array}$ & Totals \\
\hline Hand diagram positive & 10 & 7 & 17 \\
Hand diagram negative & 10 & 88 & 98 \\
Totals & 20 & 95 & 115 \\
\hline
\end{tabular}

This represents the model using the cutoff " $A$ " noted in Figure 2. Sensitivity $=50.0 \%$; specificity $=92.6 \%$; positive predictive value $=58.8 \%$; negative predictive value $=89.8 \%$.

and thus were not included in the logistic regression analysis.

If the model is based on "definite" hand diagrams, the sensitivity would be $45 \%$, and the specificity would be $96 \%$ with an ROC curve of $87 \%$. The odds ratio for a definite hand diagram (grade 1) was 4.0 (95\% CI: 91-17.2).

The hand diagram was positive in 11 cases where there was no supportive electrodiagnostic evidence of an ulnar nerve injury. In 7 of the 11 cases, the EMG was considered normal and, in the remaining 4 cases, a diagnosis of CTS was made. In no case was a positive hand diagram associated with a cervical radiculopathy or plexopathy.

\section{DISCUSSION}

In this study we have demonstrated that the ulnar hand diagram criteria have fair to good sensitivity for identifying true cases of UN and high specificity, depending on the cut-off used. Including age, gender, height, and weight in the model produced a high ROC value. This suggests that the use of a hand diagram is feasible for screening patients with hand numbness and could potentially be used in epidemiologic studies of the general population or of active workers in whom ulnar entrapments are more prevalent.

The false-positive hand diagrams were typically patients with normal electrodiagnostic studies (7 of 11). These could be patients with intermittent symptoms consistent with an UN, cervical radiculopathy, or lower trunk plexopathy, where the electrodiagnostic study was not sufficiently sensitive to make the diagnosis, or they could be true false positives. Patients with suspected thoracic outlet syndrome would fall into this category, because most have symptoms in digits 4 and 5 but normal electrodiagnostic studies. Of the remaining false positives, all the subjects had a diagnosis of CTS. It is known that the hand diagrams associated with CTS patients can be very variable and include symptoms in digits 4 and 5 . In addition, patients with CTS can have mild abnormalities of the ulnar nerve in the wrist, and this might influence their 
distribution of symptoms. Of note, none of the subjects with a positive hand diagram had electrodiagnostic findings of a C8 radiculopathy or lower trunk plexopathy.

The hand diagram was a strong predictor of the presence of an UN with an odds ratio of $>9$. BMI was not a predictor, and the literature has been inconsistent with regard to BMI and UN. Among women, lower BMI has been associated with a higher prevalence of UN, whereas it has a mixed relationship among men., ${ }^{3,5}$ Taller individuals were at a slightly higher risk for UN in this cohort, but this has not been demonstrated in other studies.

The limitations of the study include its modest sample size. A larger sample size would be more representative. Our findings are from a tertiary care center with a high percentage of positive cases. Seventeen percent of the sample had an $\mathrm{UN}$, and 44\% had CTS. This likely represents a selection bias from our referring physicians. In addition, the study was $85 \%$ female, and thus a more balanced sample would be more representative of the population. The study protocol was not sensitive to all types of UN at the wrist, and this diagnosis may have been underestimated. This could influence the sensitivity and specificity of the hand diagram, because the patients with pure motor UN at the wrist would typically lack sensory symptoms.

In conclusion, a hand diagram rating system is modestly sensitive and highly specific for UN. It can be used in a clinical or research setting for identifying subjects with a high likelihood of having an ulnar nerve compression neuropathy. The presence of CTS can be a confounder as can possible suspected TOS.

\section{REFERENCES}

1. Mondelli M, Giannini F, Ballerini M, Ginanneschi F, Martorelli E, et al. Incidence of ulnar neuropathy at the elbow in the province of Siena (Italy). J Neurol Sci 2005;15:5-10.

2. Roquelaure Y, Ha C, Leclerc A, Touranchet A, Sauteron M, Melchior $\mathrm{M}$, et al. Epidemiologic surveillance of upper-extremity musculoskeletal disorders in the working population. Arthritis Rheum 2006;55: $765-778$.

3. Descatha A, Leclerc A, Chastang JF, Roquelaure Y, and the Study Group on Repetitive Work. Incidence of ulnar nerve entrapment at the elbow in repetitive work. Scand J Work Environ Health 2004;30: 234-240.

4. Bartels RH, Verbeek AL. Risk factors for ulnar nerve compression at the elbow: a case control study. Acta Neurochir 2007;149:669-674.

5. Richardson JK, Green DF, Jamieson SC, Valentin FC. Gender, body mass and age as risk factors for ulnar mononeuropathy at the elbow. Muscle Nerve 2001;24:551-554.

6. Mondelli M, Grippo A, Mariani M, Baldasseroni A, Ansuini R, Ballerini $\mathrm{M}$, et al. Carpal tunnel syndrome and ulnar neuropathy at the elbow in floor cleaners. Neurophysiol Clin 2006;36:245-253.

7. Mondelli M, Padua L, Giannini F, Bibboò G, April I, Rossi S, et al. A self-administered questionnaire of ulnar neuropathy at the elbow. Neurol Sci 2006;27:402-411.

8. Katz JN, Stirrat CR. A self-administered hand diagram for the diagnosis of carpal tunnel syndrome. J Hand Surg 1990;15A:360-363.

9. Katz JN, Stirrat CR, Larson MG, Fossel AH, Eaton HM, Liang MH. A self-administered hand symptom diagram for the diagnosis and epidemiologic study of carpal tunnel syndrome. J Rheumatol 1990;17: 1495-1498.

10. Practice parameter for electrodiagnostic studies in ulnar neuropathy at the elbow: Summary statement. American Association of Electrodiagnostic Medicine, American Academy of Neurology, American Academy of Physical Medicine and Rehabilitation. Muscle Nerve 1999;22:408-411. 